\title{
Comparison of siRNA-mediated silencing of glycosaminoglycan synthesis genes and enzyme replacement therapy for mucopolysaccharidosis in cell culture studies
}

\author{
Izabela Chmielarz1, Magdalena Gabig-Cimińska1,2, Marcelina Malinowska1', Zyta Banecka- \\ Majkutewicz ${ }^{3}$, Alicja Węgrzyn ${ }^{4}$ and Joanna Jakobkiewicz-Banecka ${ }^{\circledR}$
}

1Department of Molecular Biology, University of Gdansk, Gdańsk, Poland; 2Laboratory of Molecular Biology, (affiliated with the University of Gdańsk), Institute of Biochemistry and Biophysics, Polish Academy of Sciences, Gdańsk, Poland; ${ }^{3}$ Department of Neurology, Medical University of Gdańsk, Gdańsk, Poland; 4Department of Microbiology, University of Szczecin, Szczecin, Poland

Cytotoxicity of laronidase (Aldurazyme ${ }^{\circ}$ ), employed in enzyme replacement therapy (ERT) for mucopolysaccharidosis type I (MPS I) and various siRNAs, tested previously in studies on substrate reduction therapy (SRT) for mucopolysaccharidoses, was tested. The enzyme did not cause any cytotoxic effects, and the siRNAs did not inhibit growth of most investigated cell lines. However, some cytotoxic effects of some tested siRNAs were observed in one MPS IIIA cell line. The efficacy of a combination of enzyme replacement therapy and siRNA-based substrate deprivation therapy was tested on three MPS I cell lines. Surprisingly, different results were obtained for different cell lines. The decrease of glycosaminoglycan storage in cells treated simultaneously with both methods was: (i) less pronounced than obtained with either of those methods used alone in one cell line, (ii) similar to that observed for enzyme replacement therapy in another cell line, and (iii) stronger than that obtained with either of the methods used alone in the third cell line. Therefore, it appears that the effects of various therapeutic methods may strongly depend on the features of the MPS cell line.

Key words: ERT, MPS, siRNA, SRT

Received: 18 November, 2012; revised: 05 December, 2012 accepted: 12 December, 2012; available on-line: 18 December, 2012

\section{INTRODUCTION}

Mucopolysacharidoses (MPS) are a group of inherited metabolic diseases characterized by accumulation of glycosaminoglycans (GAG) in lysosomes. These usually fatal disorders are caused by mutation in genes coding for enzymes involved in degradation of GAGs (Neufeld \& Muenzer, 2001). The activity deficiencies of appropriate lysosomal enzymes result in variable clinical phenotypes which include organomegaly, dysostis multiplex, decreased growth and respiratory insufficiency, and in some cases the central nervous system (CNS) is also affected (Neufeld \& Muenzer, 2001). Mucopolysaccharidoses are divided into seven distinct types, and into 11 subtypes depending on the deficient enzyme or clinical manifestation.

Since 1980 heamatopoietic stem cell transplantation (HSCT) has been used as a therapeutic approach in patients with severe MPSI (Peters et al., 1996; de Ru et al., 2011). Beneficial effects may only be achieved in children who have minimal or no symptoms from the CNS and this procedure is associated with a high incidence of graft failure, morbidity and mortality (Peters \& Steward, 2003). Enzyme replacement therapy (ERT) is another approved treatment of MPS. ERT is currently available for MPS I, MPS II and MPS VI (Beck, 2010). Introduction of human recombinant laronidase (Aldurazyme ${ }^{\circledR}$ Genzyme-Sanofi, Cambridge, MA, USA) in 2001 as the first ERT for mucopolysaccharidoses offered hope for patients and their families (Beck, 2010). Significant improvement in respiratory functions, physical capacity and reduction of organomegaly has been achieved (Kakkis et al., 2001; Wraith et al., 2004). However, neurological symptoms cannot be successfully treated due to the insufficient delivery of the enzyme through the blood-brain barrier (BBB) (Beck, 2010). Other therapeutic approaches such as small synthetic chaperones, gene therapy, stop codon read-through strategy, and substrate deprivation therapy (SDT) are under detailed investigation (Jakóbkiewicz-Banecka et al., 2007). One of them, SDT based on inhibition of GAG synthesis by genistein, is used in MPS III (Sanfilippo disease) (Piotrowska et al., 2006). Genistein (5, 7-dihydroxy-3-(4-hydroxyphenyl)-4H-1-benzopyran-4-one, also known as 4', 5, 7-trihydroxyisoflavone), an isoflavone able to cross the $\mathrm{BBB}$ is reported not only to reduce lysosomal storage, but also shows significant improvement in cognitive function and general behavior (Piotrowska et al., 2008).

Recent studies on specific inhibition of GAG synthesis by siRNA (small interfering RNA) have highlighted possible therapeutic applications for MPSs (Kaidonis et al., 2009; Dziedzic et al., 2010). Selective silencing of expression of a particular gene by specific degradation of corresponding mRNA became a standard laboratory procedure shortly after initial reports (Rana, 2007). RNA interference, first characterized in the nematode Ceanorbabiditis elegans (Fire et al., 1998), has a potential for treating wide range of diseases. Preclinical trials are being performed for treatment of amyotrophic lateral sclerosis, age-related macular degeneration, hepatitis $\mathrm{B}$ and C, HIV, hepatic cancer and solid tumor cancers (Kim \& Rossi, 2007; Snead \& Rossi, 2012). However that approach has disadvantages like immune response, oversat-

e-mail: joannaj@biotech.ug.edu.pl

Abbreviations: BBB, blood-brain barrier; CNS, central nervous system; GAG, glycosaminoglycan; HDF, human dermal fibroblast; HSCT, haematopoietic stem cell transplantation; IDUA, alpha-L-iduronidase; MPS, mucopolysaccharidosis; SDT, substrate deprivation therapy. 
uration of endogenous pathways and off-targeting (Kim \& Rossi, 2007).

The synthesis of glycosaminoglycans is initiated by formation of GAG-protein linked tetrasaccharide, GlcA $\beta 1$ $3 \mathrm{Gal} \beta 1-3 \mathrm{Gal} \beta 1-4 \mathrm{Xyl} \beta 1-O-S e r$, to the core protein. Two variants of O-xylosyltransferases (XYLT1 and XYLT2) are responsible for the initiation step (Uyama et al., 2007). In the next phase two galactose (Gal) residues are attached in reactions catalyzed by $\beta 1$,4-galactosyltransferase 7 (GALTI) and $\beta 1,3$-galactosyltransferase 6 (GALTII). $\beta 1,3$-glucuronosyltransferase (GLCATI) completes the biosynthesis by transferring a glucuronic acid (GlcA) residue to the linkage trisaccharide (Prydz \& Dalen, 2000). After the synthesis of the GAG sugar backbone, the first $N$-acetylgalactosamine (GalNAc) residue is transferred to the GlcA residue in the linkage region tetrasaccharide by $\beta 1,4-N$-acetylgalactosaminyltransferase I or II (GALNACTI or GALNACTII) activity, which is the beginning of the chain elongation process. Numerous modifications including sulfation, epimerization, and desulfation lead to production of mature and functional GAG chains (Uyama et al., 2007).

The aims of this study were: first, to determine any possible cytotoxic effects caused by siRNA and ERT; second, to assess the effectiveness of siRNA-mediated silencing of genes coding for enzymes involved in the initial steps of GAG synthesis in comparison with the enzyme replacement therapy for MPS treatment; third, to combine both approaches for possible better efficiency of treatment.

\section{MATERIALS AND METHODS}

Patients' characteristics. Skin fibroblast lines were obtained from forearm skin biopsies from mucopolysaccharidosis (MPS) type I, IIIA and IIIB patients and provided by The Children's Memorial Health Institute (Warsaw, Poland). As a control healthy human dermal fibroblast (HDF) line was purchased from Cascade Biologics (Life Technologies, Carlsbad, CA, USA). Characteristics of MPS patients genotype are presented in Table 1.

Cell lines and culture conditions. Fibroblasts were cultured in $75 \mathrm{~cm}^{2}$ culture flasks in Dulbecco's modified Eagle's medium (DMEM) supplemented with 10\% fetal bovine serum (FBS) and 1x Antibiotic Antimycotic Solution (Sigma, St Louis, MO, USA) at $37^{\circ} \mathrm{C}$ in a humidified atmosphere of $5 \% \mathrm{CO}_{2}$. Once the cells reached $90 \%$ confluency they were passaged into a 24 -well plate and the experiment was performed. For the enzyme study cells were treated with laronidase (Aldurazyme ${ }^{\circledR}$, Genzyme-Sanofi, Cambridge, MA, USA), a recombinant human $\alpha$-L-iduronidase (IDUA) at final concentrations $10,50,100 \mathrm{U} / 1$.
siRNA transfection. MPS I, IIIA and IIIB cells were seeded in triplicate into 24-well tissue culture plates, 24 $\mathrm{h}$ before transfection. Cells were transfected with Transfection Reagent HiPerFect (Qiagen GmbH, Hilden, Germany) and $25 \mathrm{nM}$ siRNAs (Applied Biosystems - Ambion, Austin, TX, USA) according to manufacturer's instruction. Pre-designed siRNAs against human genes: XYLT1 (siRNA ID\#112165, siRNA ID\#29873), XYLT2 (siRNA ID\#112168, siRNA ID\#212974), GALTI (B4GALT7) (siRNA ID\#19329, siRNA ID\#111770), GALTII (B3GALT6) (siRNA ID\#112321, siRNA ID\#112322) were the same as used and described previously by Dziedzic et al., (2010); while CS-GALNACT1 (siRNA ID\#s31518, siRNA ID\#s31519), CS-GALNACT2 (siRNA ID\#s3880, siRNA ID\#s3881) as presented in the work of Dziedzic and coworkers (2012). For further experimental procedures cell cultures were incubated for 24 or $48 \mathrm{~h}$ in standard culture conditions.

Cytotoxicity assay. The cytotoxicity assay (MT'T assay) was conducted according to the method by Mosmann, (1983), with modifications. MTT (3-(4,5-dimethylthiazol-2-yl)-2,5-diphenyltetrazolium bromide)( Sigma, St Louis, MO, USA) dissolved at $1 \mathrm{mg} / \mathrm{ml}$ in RPMI 1640 medium (Sigma, St Louis, MO, USA) was substituted for culture medium. After 2 hours of incubation at $37^{\circ} \mathrm{C}$ in humidified atmosphere of 5\% $\mathrm{CO}_{2}$, MTT solution was removed from culture plates and replaced with DMSO (dimethyl sulfoxide, Sigma, St Louis, MO, USA). Plates were incubated for 5 minutes at room temperature to dissolve formazan products. Absorbance was measured at $550 \mathrm{~nm}$ using Victor Multilabel Plate Reader (PerkinElmer Inc. - Life Technologies, Walham, MA, USA). Estimation of siRNA cytotoxic effect was conducted $24 \mathrm{~h}$ and $48 \mathrm{~h}$ after transfection procedure as described above while determination of laronidase cytotoxicity was carried out $24 \mathrm{~h}$ and $48 \mathrm{~h}$ after laronidase treatment.

Measurement of total GAGs level in cells. Fibroblasts from MPS I patients were seeded on 6-well tissue culture plates and grown for $24 \mathrm{~h}$ at $37^{\circ} \mathrm{C}$ in a humidified atmosphere of $5 \% \mathrm{CO}_{2}$. Cells were transfected with siRNA oligonucleotide ID\#19329 (GALTI) and/or treated with laronidase $(100 \mathrm{U} / \mathrm{l})$. After $48 \mathrm{~h}$, cells were washed with PBS and harvested using $0.25 \%$ trypsin-ethylene diaminetetraacetic acid solution (Sigma, St Louis, MO, USA). The pellet was digested with $0.03 \%$ papain solution from papaya latex (Sigma, St Louis, MO, USA) in $0.1 \mathrm{M}$ sodium acetate buffer for $3 \mathrm{~h}$ at $65^{\circ} \mathrm{C}$. Afterwards, a quantitative dye binding method - Blyscan (Biocolor Ltd, UK) was performed using 1,9-dimethylmethylene blue to measure sulfated GAGs. The amount of total glycosaminoglycans was standardized against DNA content measured spectrophotometrically using PicoGreen dsDNA Quantification Kit (Molecular Probes Invitrogen, Carlsbad, CA, USA).

Table 1. Characteristics of genotypes of MPS patients

\begin{tabular}{lllll}
\hline MPS type & MPS subtype & Patient ID & Mutations & Mutations description \\
\hline MPS I & Hurler & 20 & Q70X/W402X & STOP codon/STOP codon \\
& Hurler & 21 & Q70X/Q70X & STOP codon/STOP codon \\
& Not determined & 25 & delD349/delD349 & deletion/deletion \\
& Not determined & 54 & Q70X/IVS7+2T $>$ C & STOP codon/substitution T $\rightarrow$ C \\
\hline MPS III & A & 26 & R74C/G90R & Non-conservative aa substitution; destabilization of active site/ \\
& & 38 & R74C/R74C & Non-conservative aa substitution
\end{tabular}


A
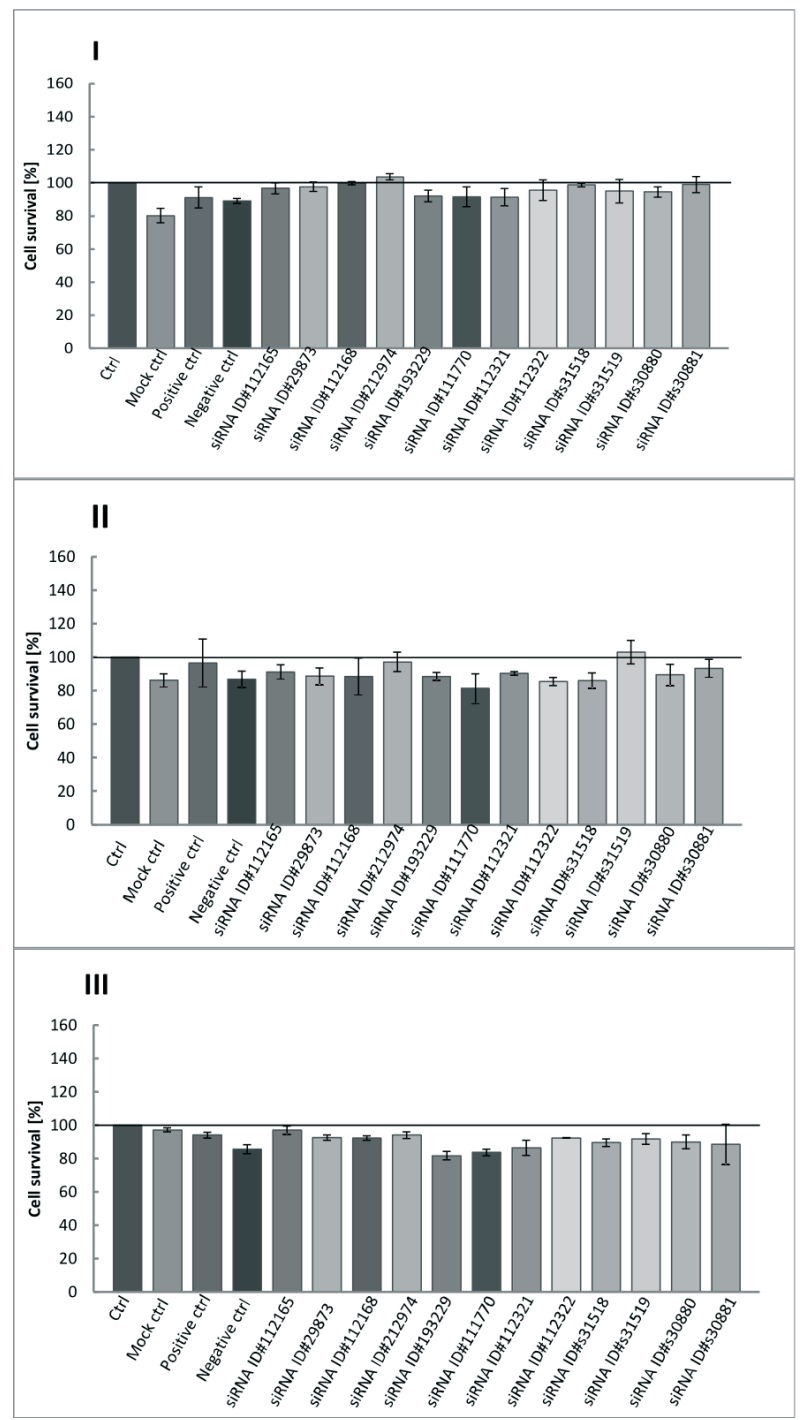

B
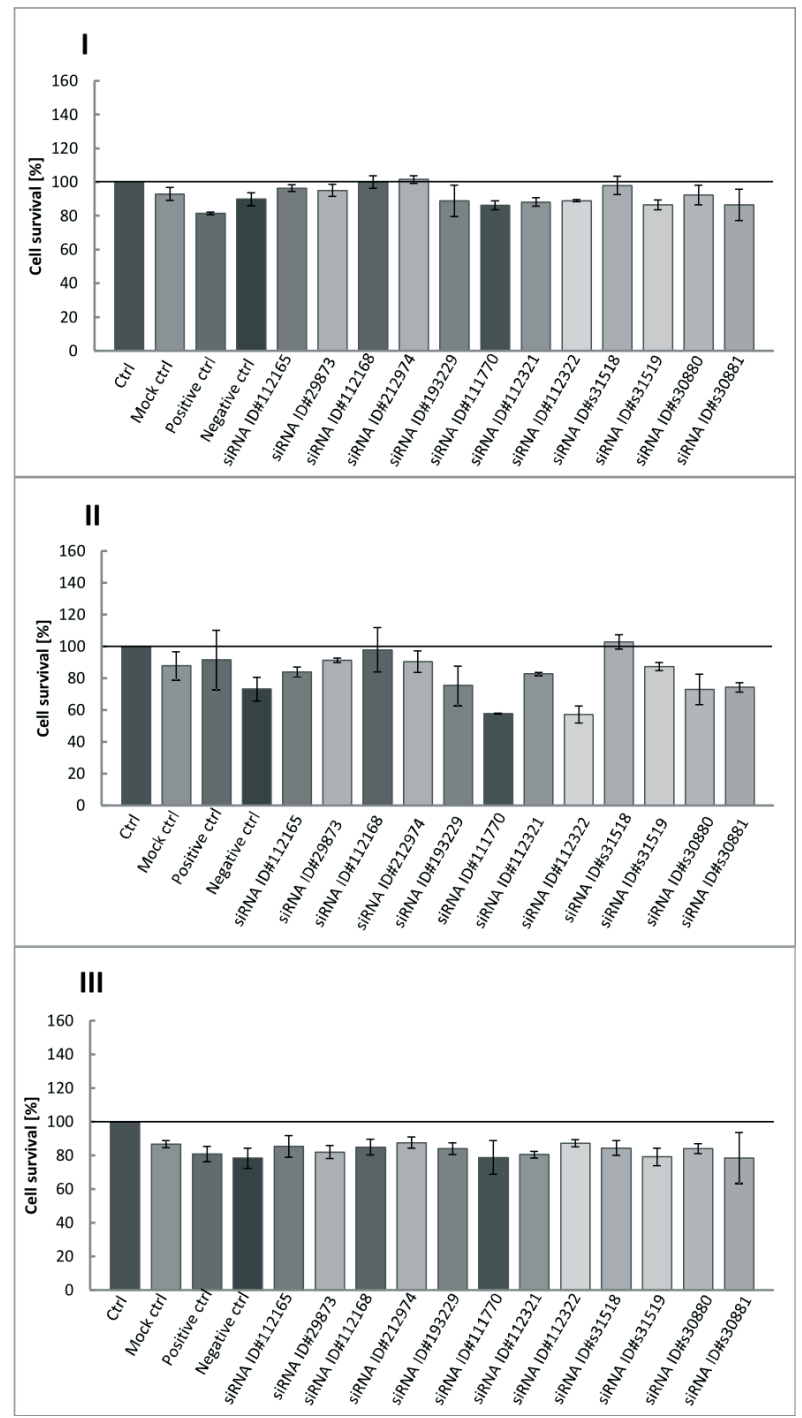

Figure 1. siRNA has no cytotoxic effect on MPS I cell lines.

Three lines of fibroblast from MPS I patients (cell line 20 - I; 21 - II; 25 - III) were transfected with the respective siRNAs: XYLT1 (siRNA ID\#112165, siRNA ID\#29873), XYLT2 (siRNA ID\#112168, siRNA ID\#212974), GALTI (B4GALT7) (siRNA ID\#19329, siRNA ID\#111770), GALTII (B3GALT6) (siRNA ID\#112321, siRNA ID\#112322), CS-GALNACT1 (siRNA ID\#s31518, siRNA ID\#s31519), CS-GALNACT2 (siRNA ID\#s3880, siRNA ID\#s3881). Panel A shows cytotoxicity effect after $24 \mathrm{~h}$ from transfection, and panel B after $48 \mathrm{~h}$. For all graphs, values are presented as means of three biological replications with corresponding SD. 'Ctrl' represents untreated cells, 'Mock ctrl' indicates conditions when only the transfection reagent was used, 'Positive ctrl' indicates transfection with siRNA against the GAPDH gene, 'Negative ctrl' represents results with AllStars Negative Control siRNA.

\section{RESULTS}

\section{siRNAs and laronidase are not cytotoxic to MPS I and MPS III fibroblasts}

Although both siRNA treatment and ERT had been tested previously for various therapeutical and biochemical parameters (see Dziedzic et al., 2010; 2012; Kakkis et al., 2001), they were not tested on in different cell lines of MPS patients. On the other hand, such testing may be important in the light of the high variability of MPS (Neufeld \& Muenzer, 2001) and possible different reactions of different cell lines, as well as different patients. Therefore, we tested the cytotoxicity of siRNA treatment on several cell lines derived from MPS I, MPS IIIA and MPS IIIB patients, and of the treatment with human re- combinant $\alpha$-L-iduronidase (laronidase, Aldurazyme ${ }^{\mathbb{R}}$ ), an enzyme that is used in ERT for MPS I.

For testing the cytotoxicity of siRNAs, we used previously described oligonucleotides (Dziedzic et al., 2010; 2012) and treated three MPS I and three MPS III cell lines of human fibroblasts. The experiments were performed for $24 \mathrm{~h}$ and $48 \mathrm{~h}$ for each cell line. No cytotoxicity was observed for any tested MPS I cell lines and for any tested siRNAs (Fig. 1). Similar results were obtained in experiments with two MPS IIIA and one MPS IIIB cell lines, however, one evident case of cytotoxicity was found in one of the MPS IIIA cell lines (Fig. 2, panel III-B). Moreover, several cases of weak cytotoxic effects were also observed in the same cell line. All these cytotoxic effects occurred after 48 h (Fig. 2). These results indicate that siRNA treatment is generally not cyto- 
A
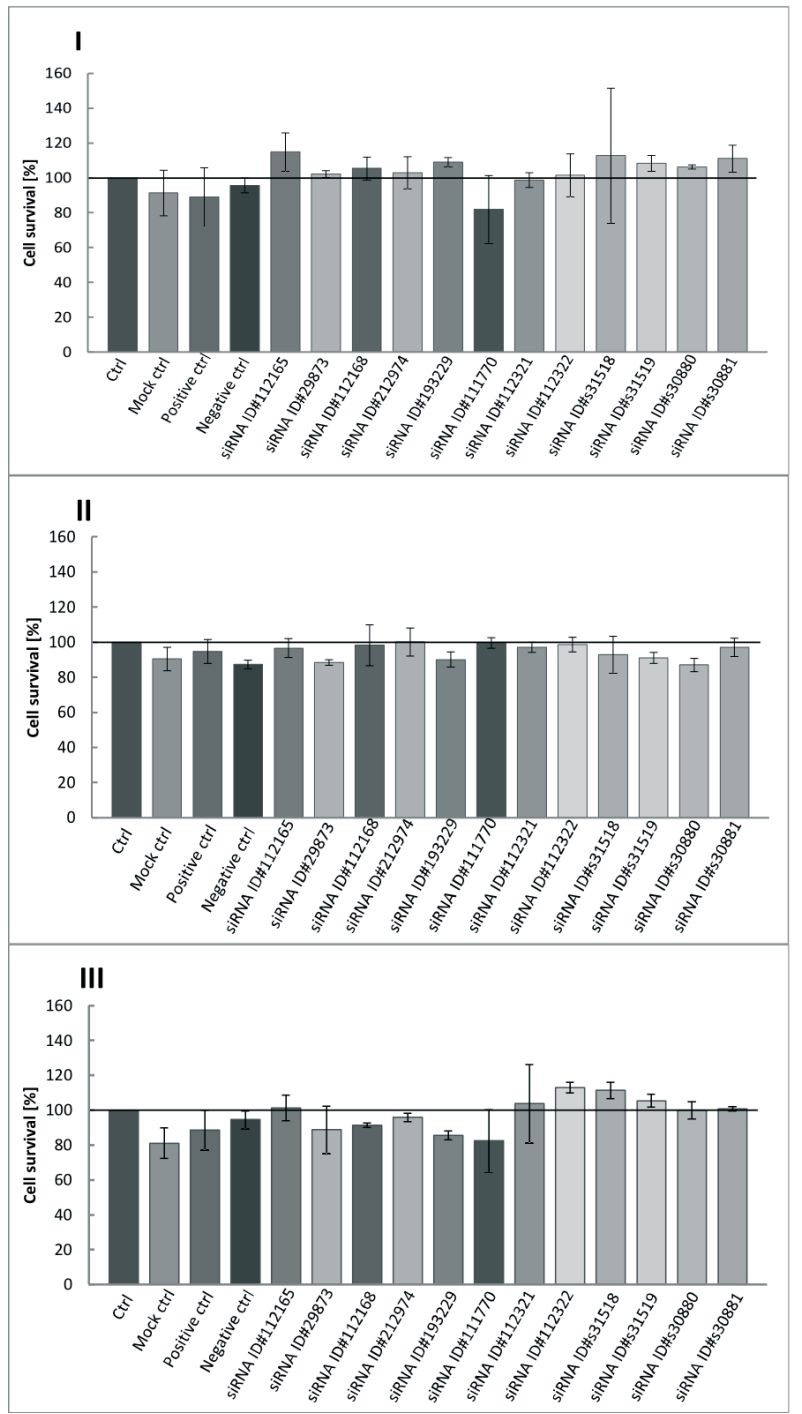

B
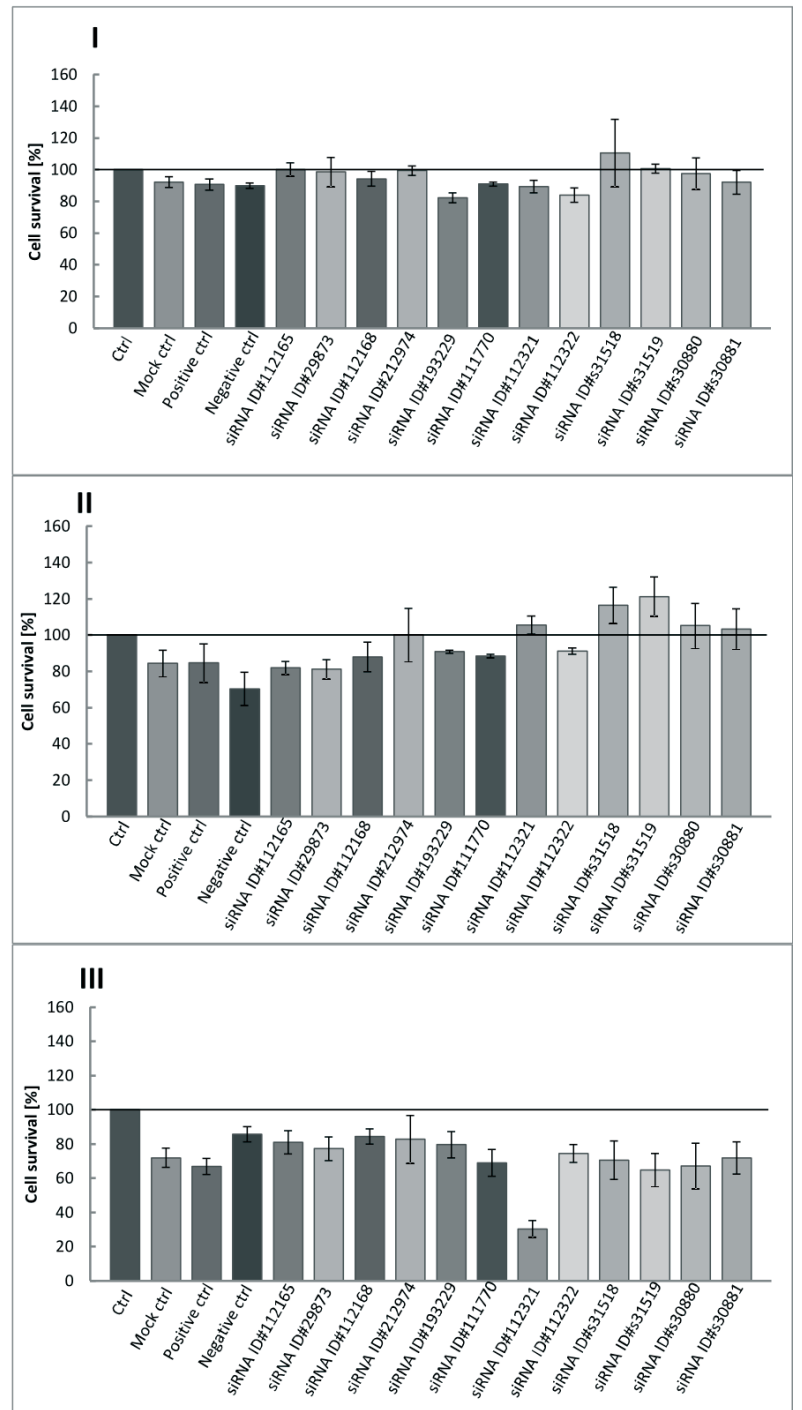

Figure 2. Cytotoxic effects of siRNAs to MPS IIIA and B cell lines.

Three lines of fibroblast from MPS III patients (cell line $27-\mathrm{I} ; 38$ - II; 26 - III) were transfected with the respective siRNAs: XYLT1 (siRNA ID\#112165, siRNA ID\#29873), XYLT2 (siRNA ID\#112168, siRNA ID\#212974), GALTI (B4GALT7) (siRNA ID\#19329, siRNA ID\#111770), GALTII (B3GALT6) (siRNA ID\#112321, siRNA ID\#112322), CS-GALNACT1 (siRNA ID\#s31518, siRNA ID\#s31519), CS-GALNACT2 (siRNA ID\#s3880, siRNA ID\#s3881). Panel A shows cytotoxicity effects after $24 \mathrm{~h}$ from transfection, panel B, effects after $48 \mathrm{~h}$. For all graphs values are presented as the means of three biological repetition with corresponding SD. 'Ctrl' represents untreated cells, 'Mock ctrl' indicates conditions when only the transfection reagent was used, 'Positive ctrl' indicates transfection with siRNA against the GAPDH gene, 'Negative ctrl' represents results with Allstars Negative Control siRNA.

toxic for MPS cells, however, sporadic effects may occur in cells of particular patients.

Unlike for the siRNA treatment, no cytotoxic effects occurred in any of the MPS I cell lines treated with laronidase (Fig. 3). These results confirm that ERT for MPS I is a generally safe treatment in as regards its cytotoxicity.

\section{Effects of ERT, siRNA and combined treatments on GAG storage in MPS I cells}

We aimed to compare the effects of different treatment methods on the reduction of GAG storage in various cell lines of MPS I. Three MPS I cell lines were treated with laronidase, siRNA or their combination for 48 h. In control experiments, no significant effects of
ERT, siRNA treatment or both methods used together on GAGs level was observed in wild-type human fibroblasts (results not shown).

As expected, ERT was more effective than siRNA treatment in reduction of GAGs storage in all tested cell lines (Fig. 4). However, different results were obtained for different cell lines when a combination of these two therapeutic approaches was employed. Depending on the cell line the decrease in GAG storage in cells treated simultaneously with both methods was less pronounced than that obtained with either method used alone (Fig. 4A), similar to that observed in ERT (Fig. 4B), or more pronounced than that obtained with either method alone (Fig. 4C). Therefore, it appears that effects of various 

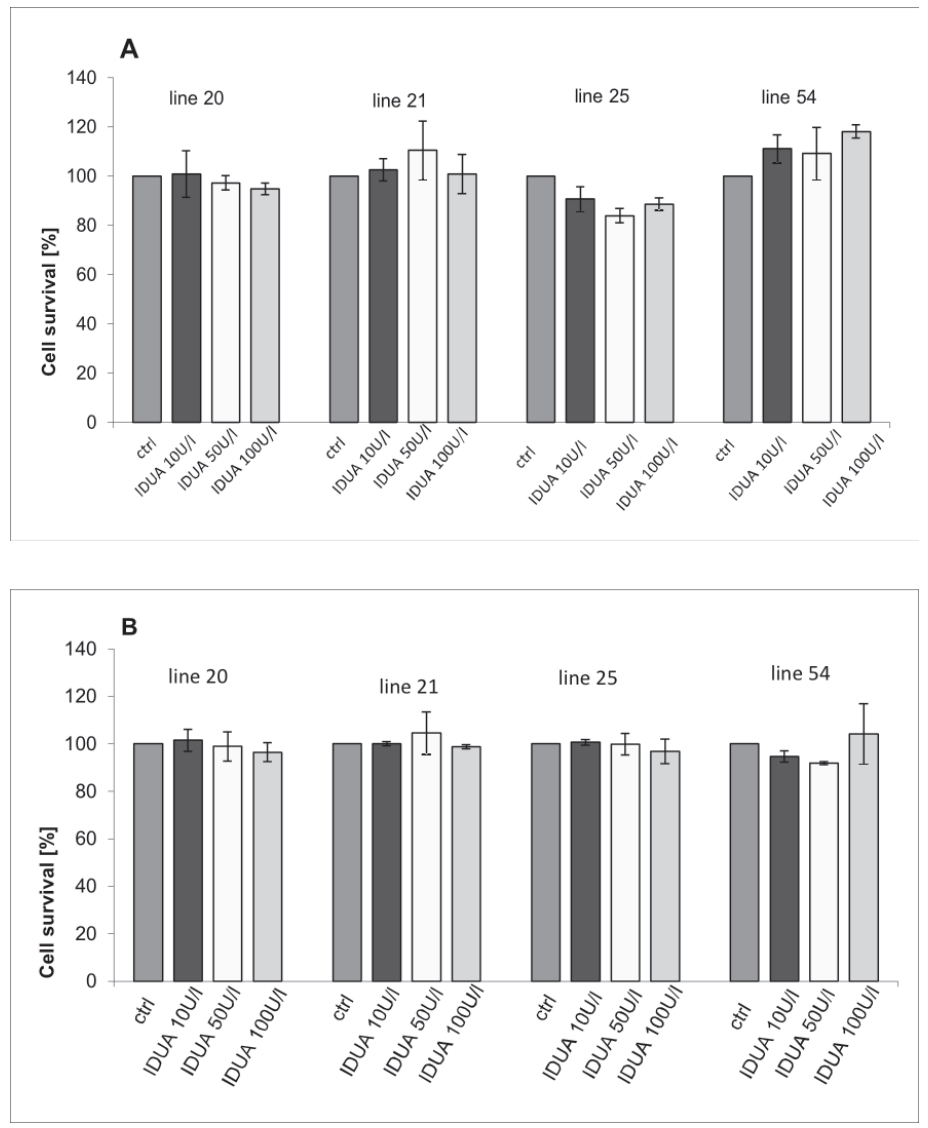

Figure 3. Laronidase has no cytotoxic effect on MPS I cell lines.

Four cell lines from MPS I patients (cell lines: 20, 21, 25, 54) were treated with appropriate concentrations of laronidase (IDUA) (10, 50, $100 \mathrm{U} / \mathrm{I})$, and after 24 $\mathrm{h}(\mathbf{A})$ or $48 \mathrm{~h}(\mathbf{B})$, cytotoxicity was assessed using the MTT assay. therapeutic methods may strongly depend on the cell line studied.

\section{DISCUSSION}

Although various therapeutic methods for MPS are currently being considered and tested, none of the available treatments is sufficiently effective to cure those severe diseases (Jakóbkiewicz-Banecka et al., 2007; Beck, 2010). Therefore, there is a need for the development of new therapies or combinations of already known.

In this report we tested two treatment procedures for MPS, ERT and substrate deprivation therapy based on the use of siRNA. In our approach we used several different cell lines derived from patients suffering from MPS I or MPS III. Perhaps surprisingly, we found that neither laronidase (Aldurazyme ${ }^{\circledR}$ ) is cytotoxic for any of the MPS I cell lines tested, nor siRNAs are cytotoxic for most of the MPS I and MPS III cell lines tested. However some cytotoxicity of several siRNAs was observed for one MPS IIIA cell line. As this effect was also observed for the Mock control, the cell line sensitivity to the transfection agent rather than siRNA-dependent cytotoxicity should be considered in this case. We assume this could be an important discovery, as it might indicate that a generally safe therapeutic procedure for MPS can cause adverse effects in some patients. The siRNA-sensitive cell line derived from a patient suffering from MPS IIIA (Table 1, patient no. 26). This patient is a complex heterozygote, but one could not predict the unusual reaction to siRNA on the basis of such a genotype. Thus, we as-
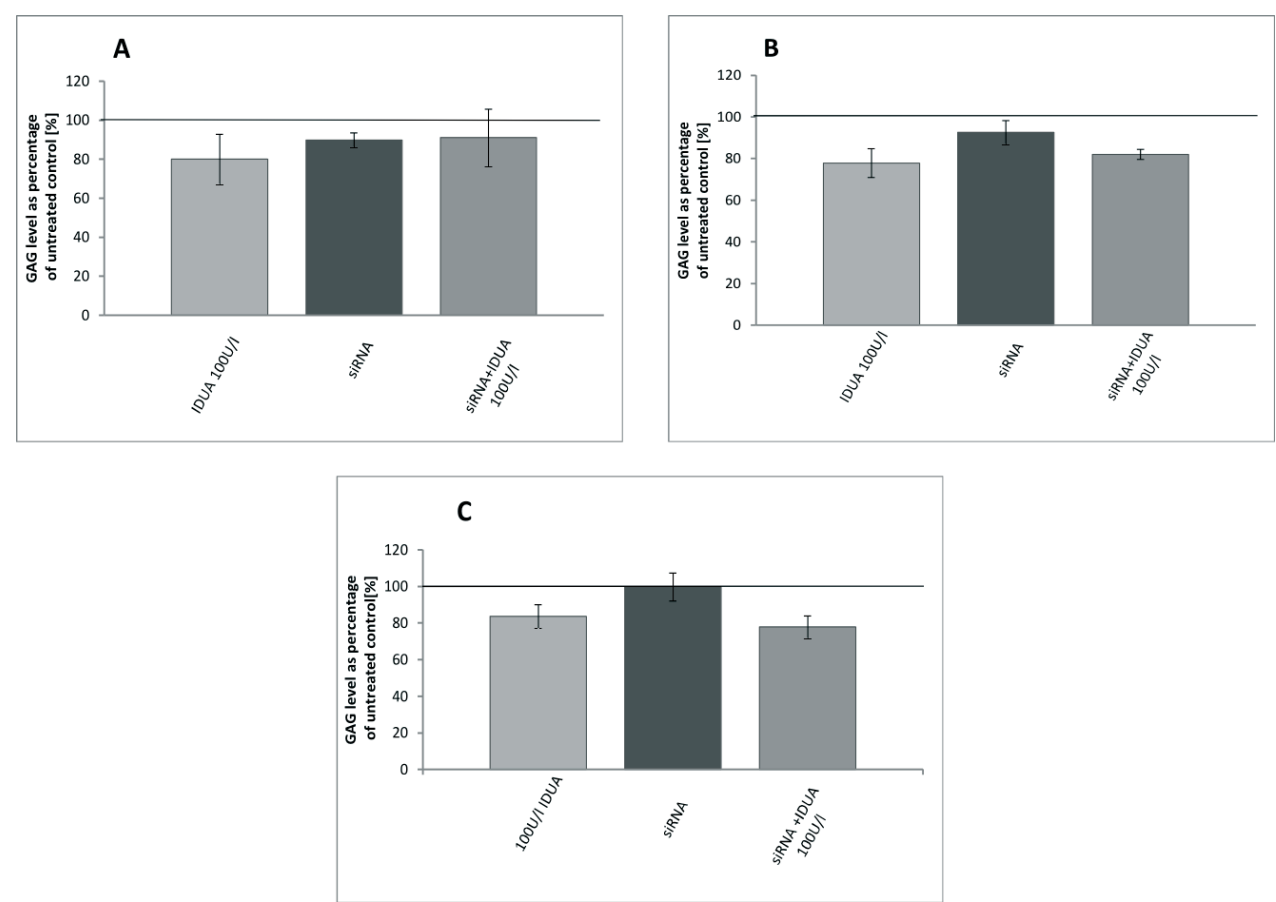

Figure 4. Effects of combined siRNA and laronidase treatment on MPS I cell lines.

Measurement of total GAGs was performed on three MPS I cell lines $48 \mathrm{~h}$ after siRNA transfection or/and laronidase (IDUA) treatment, according to procedures described in Methods. The results are presented as the percentage of untreated control \pm S.D. Panel A shows results of experiments with cell line 54, panel B, cell line 21; and panel C, cell line 20. 
sume that the genetic background of this patient may be responsible for the siRNA sensitivity.

One of the therapeutic approaches that could potentially result in efficacy higher than that achieved in trials performed to date is combination of various treatment procedures. Therefore, we tested the effects of a combination of ERT and treatment with siRNA on GAG storage in three different MPS I cell lines. Surprisingly, the results varied significantly between these cell lines. The combined therapy was either less effective, of similar efficiency or more effective than ERT used alone, depending on the cell line (Fig. 4). Surprisingly, the highest efficacy was observed in the cell line of the genotype Q70X/W402X, i.e. bearing two nonsense mutation in the IDUA gene (Fig. 4C). One might predict that the substrate deprivation therapy with the use of siRNA should be of the highest efficiency in cells containing some residual activity of the deficient enzyme, and of the lowest efficiency in the case of cells unable to produce full-length enzyme. This is clearly not the case. Furthermore, the second cell line characterized by two null mutations responded differentially to the combined therapy (Fig. 4B), and the only cells potentially producing an $\alpha$-L-iduronidase variant with some residual activity revealed the weakest efficiency in reduction of GAG levels when treated simultaneously with ERT and siRNA (Fig. 4A).

In summary, the results presented in this report indicate that laronidase (Aldurazyme ${ }^{\circledR}$ ) and siRNAs do not cause cytotoxicity in most MPS cell lines, however, some cytotoxic effects could occur in particular cell lines. Moreover, the efficacy of a combination of ERT and the use of siRNA depends on specific features of each cell line, thus, the combined therapy might potentially result in various effects in different patients.

\section{Acknowledgments}

This work was supported by the Foundation for Polish Science Team Programme co-financed by the EU European Regional Development Fund (grant no. TEAM/2008-2/7).

\section{REFERENCES}

Beck M (2010) Therapy for lysosomal storage disorders. IUBMB Life 62: 33-40.

de $\mathrm{Ru}$ M, Boelens JJ, Das AM, Jones SA, van der Lee JH, Mahlaoui N, Mengel E, Offringa M, O’Meara A, Parini R, Rovelli A, Sykora KW, Valayannopoulos V, Vellodi A, Wynn RF, Wijburg FA (2011) Enzyme replacement therapy and/or hematopoietic stem cell transplantation at diagnosis in patients with mucopolysaccharidosis type I: results of a European consensus procedure. Orphanet J Rare Dis 6: 55 .

Dziedzic D, Narajczyk M, Gabig-Cimińska M, Jakóbkiewicz-Banecka J (2012) Simultaneous siRNA-mediated silencing of pairs of genes coding for enzymes involved in glycosaminoglycan synthesis. Acta Biochim Pol 59: 293-298.
Dziedzic D, Wegrzyn G, Jakóbkiewicz-Banecka J (2010) Impairment of glycosaminoglycan synthesis in mucopolysaccharidosis type IIIA cells by using siRNA: a potential therapeutic approach for Sanfilippo disease. Eur I Hum Genet 18: 200-205.

Fire A, Xu S, Montgomery MK, Kostas SA, Driver SE, Mello CC (1998) Potent and specific genetic interference by double-stranded RNA in Caenorbabditis elegans. Nature 19: 806-811.

Hobbs JR, Hugh-Jones K, Barrett AJ, Byrom N, Chambers D, Henry K, James DC, Lucas CF, Rogers TR, Benson PF, Tansley LR, Patrick AD, Mossman J, Young EP (1981) Reversal of clinical features of Hurler's disease and biochemical improvement after treatment by bone-marrow transplantation. Lancet 2: 709-712.

Jakóbkiewicz-Banecka J, Wegrzyn A, Wegrzyn G (2007) Substrate deprivation therapy: a new hope for patients suffering from neuronopathic forms of inherited lysosomal storage diseases. I Appl Genet 48: 383-388.

Kaidonis X, Liaw W.C, Roberts AD, Ly M, Anson D, Byers S (2010) Gene silencing of EXTL2 and EXTL3 as a substrate deprivation therapy for heparan sulfate storing mucopolysaccharidoses. Eur $J$ Hum Genet 18: 194-199.

Kakkis ED, Muenzer J, Tiller GE, Waber L, Belmont J, Passage M, Izykowski B, Phillips J, Doroshow R, Walot I, Hoft R, Neufeld EF (2001) Enzyme-replacement therapy in mucopolysaccharidosis I. N Engl J Med 344: 182-188.

Kim DH, Rossi JJ (2007) Strategies for silencing human disease using RNA interference. Nat Rev Genet 8: 173-184.

Mosmann T (1983) Rapid colorimetric assay for cellular growth and survival: Application to proliferation and cytotoxicity assays. $\mathrm{I} \mathrm{Im-}$ munol Methods 65: 55-63.

Neufeld EF, Muenzer J (2001) The mucopolysaccharidoses. In The Metabolic and Molecular Bases of Inberited Disease. Scriver CR et al., eds pp 3421-3452. McGraw-Hill Co. New York.

Peters C, Balthazor M, Shapiro EG, King RJ, Kollman CC, Hegland JD, Henslee-Downey J, Trigg ME, Cowan MJ, Sanders J, Bunin N, Weinstein H, Lenarsky C, Falk P, Harris R, Bowen T, Wiliams TE, Grayson GH, Warkentin P, Sender L, Cool VA, Critten M, Packman S, Kaplan P, Lockman LA, Anderson J, Krivit W, Dusenbery $\mathrm{K}$, Wagner J (1996) Outcome of unrelated donor bone marrow transplantation in 40 children with Hurler syndrome. Blood vol 87: 4894-4902.

Peters C, Steward CG. (2003) Hematopoietic cell transplantation for inherited metabolic diseases: an overview of outcomes and practice guidelines. Bone Marrow Transplant 31: 229-239.

Piotrowska E, Jakobkiewicz-Banecka J, Baranska S, Tylki-Szymanska A, Czartoryska B, Wegrzyn A, Wegrzyn G (2006) Genistein-mediated inhibition of glycosaminoglycan synthesis as a basis for gene expression-targeted isoflavone therapy for mucopolysaccharidoses. Eur J Hum Genet 14: 846-852.

Piotrowska E, Jakóbkiewicz-Banecka J, Tylki-Szymańska A, Czartoryska B, Węgrzyn A, Węgrzyn G (2008) Correlation between severity of mucopolysaccharidosis and combination of residua enzyme activity and efficiency of glycosaminoglycan synthesis. Acta Paediatr 98: $743-749$.

Prydz K, Dalen KT (2000) Synthesis and sorting of proteoglycans. J Cell Sci 113: 193-205.

Rana TM (2007) Illuminating the silence: understanding the structure and function of small RNAs. Nat Rev Mol Cell Biol 8: 23-36.

Snead NM, Rossi JJ. (2012) RNA Interference trigger variants: getting the most out of RNA for RNA interference-based therapeutics. Nucleic Acids Ther 22: 139-146.

Uyama T, Kitagawa H, Sugahara K (2007) Biosynthesis of glycosaminoglycans and proteoglycans. In Comprehensive Glycoscience. Vol. 1, pp 79-99. Elsevier.

Wraith JE, Clarke LA, Beck M, Kolodny EH, Pastores GM, Muenzer J, Rapoport DM, Berger KI, Swiedler SJ, Kakkis ED et al. (2004) Enzyme replacement therapy for mucopolysaccharidosis I: a randomized, double-blinded, placebo-controlled, multinational study of recombinant human alpha-L-iduronidase (laronidase). J Pediatr 144: 581-588. 